\title{
Problems in diabetic retinopathy treatment and management during the COVID-19 pandemic
}

\author{
Hasan Öncül ${ }^{1}$ and Mehmet Fuat Alakuş ${ }^{1}$ \\ ${ }^{1}$ Diyarbakir Gazi Yasargil Training and Research Hospital
}

November 19, 2020

\begin{abstract}
Purpose: The aim of this study is to determine the problems occurring during the COVID-19 pandemic period in patients treated with intravitreal (IV) injection for diabetic retinopathy, and to provide recommendations for treatment management in these patients. Methods: Twenty-nine eyes of 17 patients were included in this prospective, observational study. The frequency of hospital visits, treatments performed, and detailed ophthalmological examination findings, including optical coherence tomography findings and glycated haemoglobin (HbA1c) values, were recorded in the period before the COVID-19 pandemic. During the COVID-19 pandemic period, the detailed ophthalmological examination findings and HbA1c values were noted after the patients who had delayed their routine control time (>90 days) applied to the hospital. New treatments were planned according to the current situation. Results: Seventeen patients who were diagnosed with diabetic macular edema were included in the study; 10 were female $(58.8 \%)$ and seven were male $(41.2 \%)$. The mean time interval between following visits was $45.52 \pm 5.85$ days during the pre-pandemic period, but it increased to $110.41 \pm 13.47$ days during the COVID-19 pandemic period $(\mathrm{p}<.001)$. Visual acuity (LogMAR) was $0.44 \pm 0.36$ in the pre-pandemic period and $0.76 \pm 0.48$ during the COVID-19 pandemic period ( $\mathrm{p}=.003$ ). Central macular thickness was $300.10 \pm 85.56 \mu \mathrm{m}$ in the pre-pandemic period and it increased to $387.10 \pm 144.48$ $\mu \mathrm{m}$ during the COVID-19 pandemic period $(\mathrm{p}=.007)$. In three patients, complications of diabetic retinopathy that could not be cured by medical treatment developed and surgical treatment was recommended. Conclusions: Delays in the treatment of diabetic retinopathy may cause permanent impairment in visual functions. The COVID-19 pandemic has caused an increase in the hospital visit intervals of patients, and this situation has resulted in disruptions in the follow-up and treatment of patients with diabetic retinopathy. Alternative diagnosis and treatment practices are needed in order to manage these and similar processes smoothly.
\end{abstract}

\section{Hosted file}

IJCP DRP COV\selectlanguage\{polish\}I\selectlanguage\{english\}D 19 MA \selectlanguage\{polish\}I \selectlangu available at https://authorea.com/users/377137/articles/493923-problems-in-diabeticretinopathy-treatment-and-management-during-the-covid-19-pandemic

\section{Hosted file}

DRP COV\selectlanguage\{polish\}I\selectlanguage\{english\}D 19 TABLE 1.pdf available https://authorea.com/users/377137/articles/493923-problems-in-diabetic-retinopathytreatment-and-management-during-the-covid-19-pandemic

\section{Hosted file}

DRP COV\selectlanguage\{polish\}I\selectlanguage\{english\}D 19 TABLE 2.pdf available https://authorea.com/users/377137/articles/493923-problems-in-diabetic-retinopathytreatment-and-management-during-the-covid-19-pandemic 

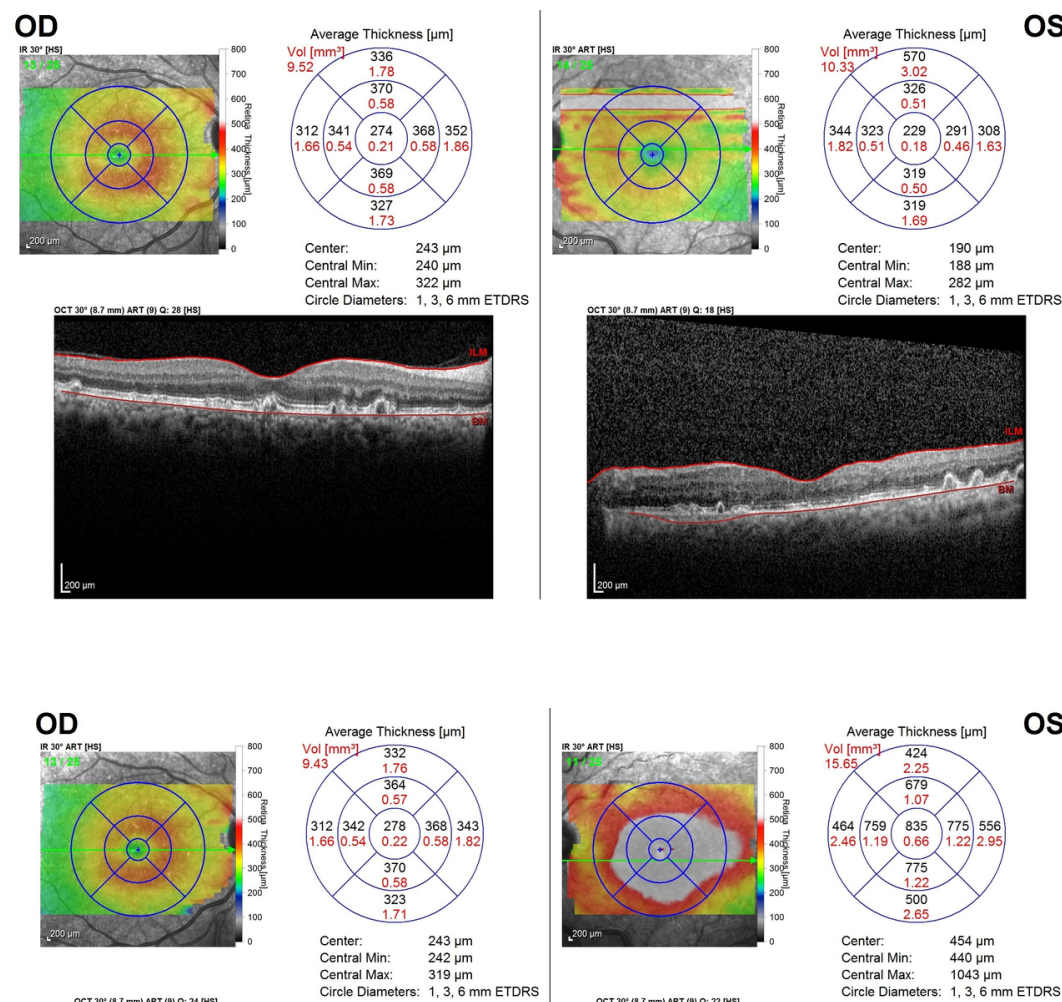

Average Thickness Im

os
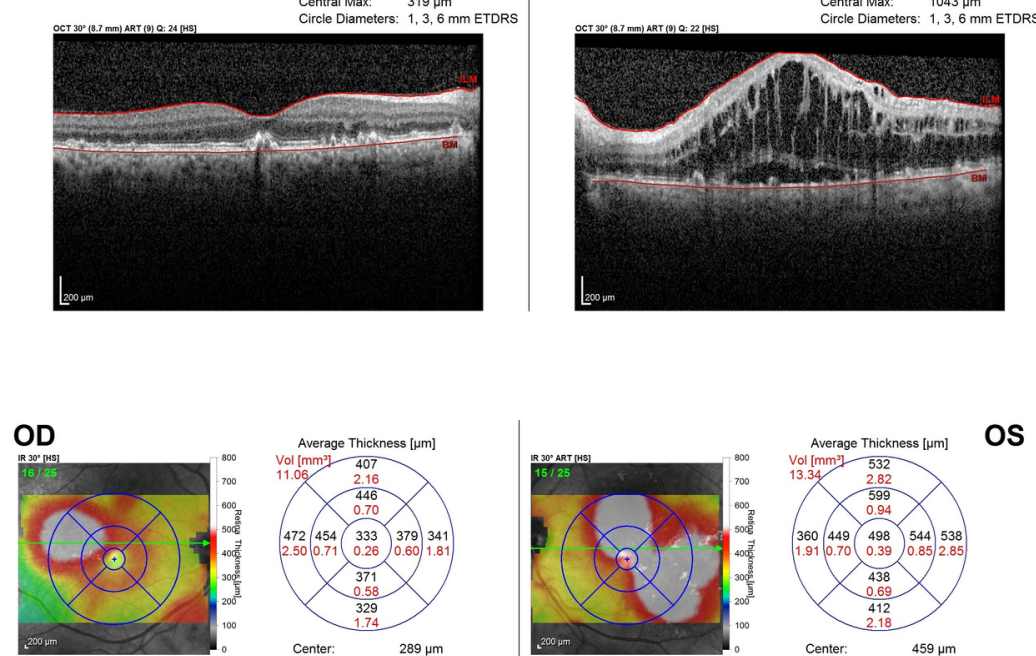

$\begin{array}{ll}\text { Center: } & 289 \mu \mathrm{m} \\ \text { Central Min: } & 272 \mu \mathrm{m} \\ \text { Central Max: } & 443 \mathrm{um}\end{array}$

Circle Diameters: $1,3,6 \mathrm{~mm}$ ETDRS
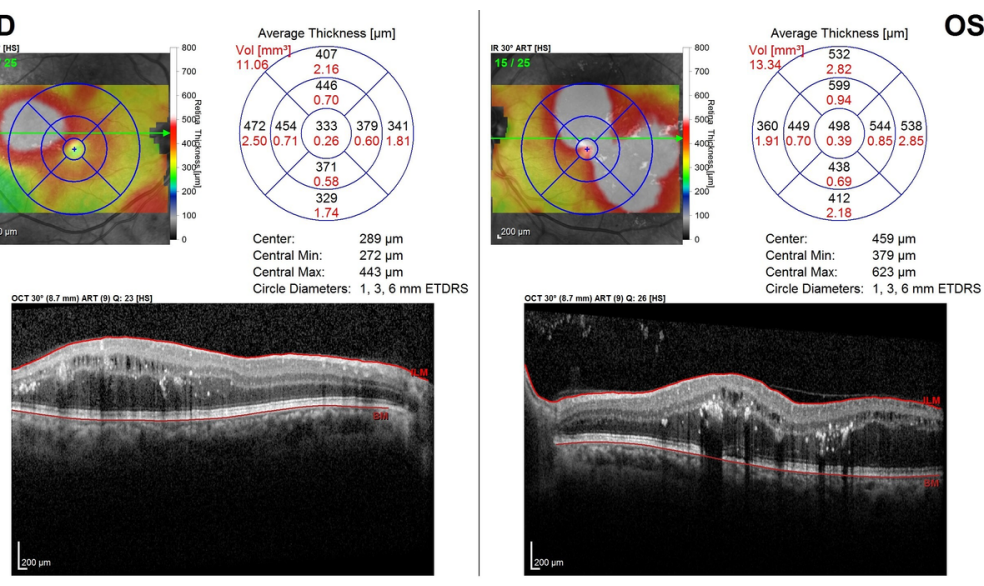

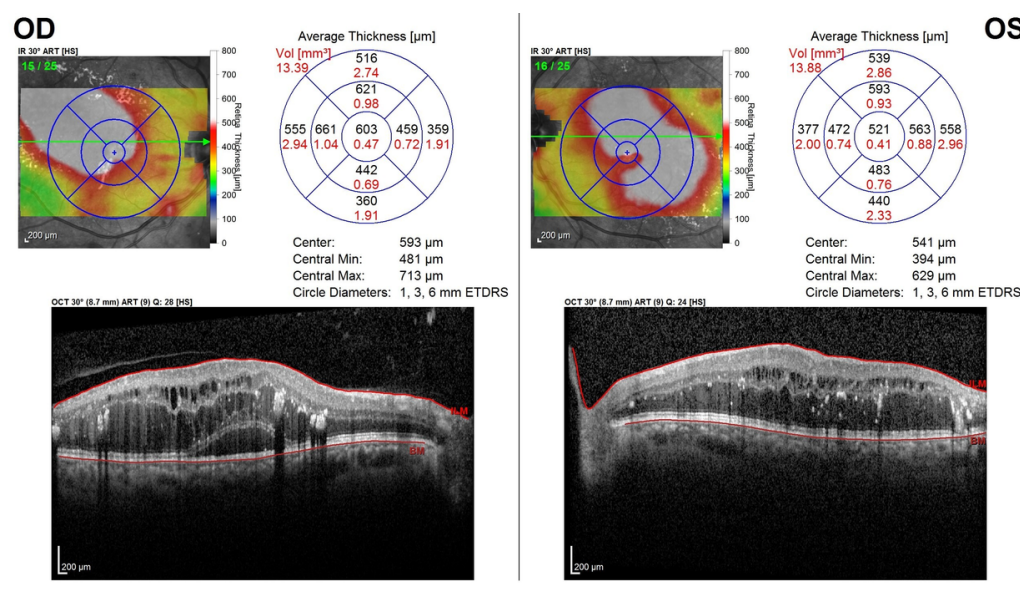